\title{
Discrete Affinity Matrices for Automatic Face Labeling
}

\author{
Pooja Gulmire ${ }^{1}$, Deepak Dharrao ${ }^{2}$ \\ ${ }^{1}$ Savitribai Phule Pune University, Indira College of Engineering and Management, Pune, Maharashtra, India \\ ${ }^{2}$ Professor, Savitribai Phule Pune University, Indira College of Engineering and Management, Pune, Maharashtra, India
}

\begin{abstract}
Given a gathering of pictures, where every picture contains number of confronts and is connected with a couple names in the comparing inscription, the objective of face naming is to induce the right name for every face. In this task, we propose two new systems to viably tackle this issue by taking in two discriminative proclivity grids from these feebly marked pictures. Firstly we propose another system called regularized low-rank representation by adequately using pitifully regulated data to take in a low-rank recreation coefficient framework while find out about different subspace structures of the information. In particular, by acquainting an exceptionally planned regularizer with the low-rank representation technique, we punish the comparing recreation coefficients identified with the circumstances where a face is reproduced by using so as to utilize face pictures from different subjects or itself. With the surmised reproduction coefficient lattice, a discriminative proclivity network can be gotten. In addition, we additionally add to another separation metric learning strategy called equivocally regulated auxiliary metric using so as to learn pitifully administered data to look for a discriminative separation metric. Henceforth, another discriminative proclivity framework can be gotten utilizing the likeness lattice (i.e., the piece network) in view of the Mahalanobis separations of the information. Watching that these two liking frameworks contain integral data, we further consolidate them to get an intertwined liking lattice, in light of which we build up another iterative plan to surmise the name of every face. Exhaustive analyses show the viability of our methodology.
\end{abstract}

Keywords: Face detection, affinity matrix, human sensing, boosting, low rank representation.

\section{Introduction}

Image processing is a method to perform some operations on an image, in order to get an enhanced image or to extract some useful information from it. It is a type of signal processing in which input is an image and output may be image or characteristics/features associated with that image.

In this paper, we concentrate on naturally clarifying countenances in pictures taking into account the vague supervision from the related inscriptions gives. Some preprocessing steps should be directed before performing face naming. In particular, faces in the pictures are consequently identified utilizing face finders, and names in the subtitles are naturally extricated utilizing a name element indicator. Here, the rundown of names showing up in an inscription is meant as the applicant name set. Indeed, even after effectively performing these preprocessing steps, programmed face naming is still a testing assignment. The countenances from the same subject may have diverse appearances due to the varieties in stances, enlightenments, and expressions. Additionally, the hopeful name set may be boisterous and inadequate, so a name may be specified in the inscription, however the relating face may not show up in the picture, and the right name for a face in the picture may not show up in the comparing subtitle. Each recognized face (counting erroneously identified ones) in a picture must be explained utilizing one of the names as a part of the competitor name set or as invalid, which shows that the ground-truth name does not show up in the inscription.

In social networking websites (e.g., Facebook), photograph sharing sites (e.g., Flickr) and news sites (e.g., BBC), a picture that contains different appearances can be connected with an inscription determining who is in the photo. Case in point, different confronts may show up in a news photograph with a subtitle that quickly portrays the news. In addition, in TV serials, motion pictures, and news features, the confronts might likewise show up in a feature cut with scripts. In the writing, a couple of strategies were created for the face naming issue.

\subsection{Related Work}

Robust real-time face detection

This paper describes a face detection framework that is capable of processing images extremely rapidly while achieving high detection rates. There are three key contributions. The first is the introduction of a new image representation called the "Integral Image" which allows the features used by our detector to be computed very quickly. The second is a simple and efficient classifier which is built using the AdaBoost learning algorithm (Freund and Schapire, 1995) to select a small number of critical visual features from a very large set of potential features. The third contribution is a method for combining classifiers in a "cascade" which allows background regions of the image to be quickly discarded while spending more computation on promising face-like regions. A set of experiments in the domain of face detection is presented. The system yields face detection performance comparable to the best previous systems (Sung and Poggio, 1998; Rowley et al., 1998; Schneiderman and Kanade, 2000; Roth et al., 2000). Implemented on a conventional desktop, face detection proceeds at 15 frames per second.[1]

A graph based approach for naming faces in news photos.

A method to associate names and faces for querying people in large news photo collections. On the assumption that a person's face is likely to appear when his/her name is mentioned in the caption, first all the faces associated with 


\section{International Journal of Science and Research (IJSR) \\ ISSN (Online): 2319-7064}

Index Copernicus Value (2013): 6.14 | Impact Factor (2014): 5.611

the query name are selected. Among these faces, there could be many faces corresponding to the queried person in different conditions, poses and times, but there could also be other faces corresponding to other people in the caption or some non-face images due to the errors in the face detection method used. However, in most cases, the number of corresponding faces of the queried person will be large, and these faces will be more similar to each other than to others. In this study, we propose a graph based method to find the most similar subset among the set of possible faces associated with the query name, where the most similar subset is likely to correspond to the faces of the queried person. When the similarity of faces are represented in a graph structure, the set of most similar faces will be the densest component in the graph. We represent the similarity of faces using SIFT descriptors. The matching interest points on two faces are decided after the application of two constraints, namely the geometrical constraint and the unique match constraint. The average distance of the matching points are used to construct the similarity graph. The most similar set of faces is then found based on a greedy densest component algorithm. The experiments are performed on thousands of news photographs taken in real life conditions and, therefore, having a large variety of poses, illuminations and expressions[2].

\section{Robust subspace segmentation by low-rank representation}

In this paper low-rank representation (LRR) to segment data drawn from a union of multiple linear subspaces. Given a set of data vectors, LRR seeks the lowest- rank representation among all the candidates that represent all vectors as the linear com- bination of the bases in a dictionary. Unlike the well-known sparse representation (SR), which computes the sparsest representation of each data vector individually, LRR aims at nding the lowest-rank representation of a collection of vectors jointly. LRR better captures the global structure of data, giving a more e®ective tool for robust subspace segmentation from corrupted data. Both the oretical and experimental results show that LRR is a promising tool for subspace segmentation[3].

\section{Cross-media alignment of names and faces}

This paper experiments on aligning names and faces as found in images and captions of online news websites. Developing accurate technologies for linking names and faces is valuable when retrieving or mining information from multimedia collections. We perform exhaustive and systematic experiments exploiting the symmetry between the visual and textual modalities. This leads to different chemes for assigning names to the faces, assigning faces to the names, and establishing name-face link pairs. On top of that, we investigate generic approaches to the use of textual and visual structural information to predict the presence of the corresponding entity in the other modality. The proposed methods are completely unsupervised and are inspired by methods for aligning phrases andwords in texts of different languages developed for constructing dictionaries for machine translation. The results are competitive with state of the art performance on the "Labeled Faces in the Wild" dataset in terms of recall values, now reported on the complete dataset, include excellent precision values, and show the value of text and image analysis for identifying the probability of being pictured or named in the alignment process[4].

\subsection{Proposed Methodology}

A Proposed Structure has a software perplex, the server needs to execute three modules as following.

The third contribution is a method of "cascade" which allowscombining classifiers in a background regions of the imageto

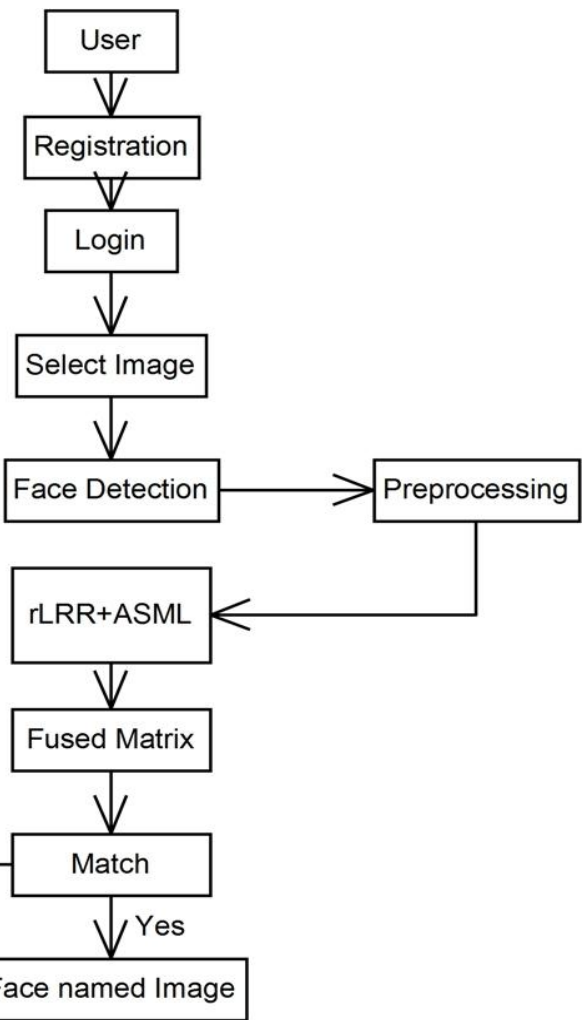




\section{International Journal of Science and Research (IJSR) \\ ISSN (Online): 2319-7064}

Index Copernicus Value (2013): 6.14 | Impact Factor (2014): 5.611

1) Face Detection

It is done using three contributions. First is introduction of new image representation called the "Integral Image" which allows features use by detector to be computed very quickly. The second is simple and efficient classifier which is built using the AdaBoostlearning algorithms to select small number of critical visual featurefrom very large set of potential features.bequickly discarded while spending more computation on promising face like region.

2) Face Naming

In this area, propose another methodology for programmed face naming with inscription based supervision and formally present the issue and definitions, trailed by the presentation of proposed methodology. In particular,here learn two discriminative fondness networks by adequately using the uncertain marks, and perform face naming in view of the intertwined partiality framework. For automatic face naming is done using proposed methodologies rLRR and ASML for getting the two liking lattices separately.

3) Camera interface

In this project we are using camera for taking images to store in database and also for searching image from database we are taking image for searching.Camera Link is a communication interface for vision applications. The interface extends the base technology of Channel Link to provide a specification more useful for vision applications.

\section{Conclusion}

In this paper, we have proposed another plan for face naming with subtitle based supervision, in which one picture that may contain numerous countenances is connected with an inscription determining just who is in the picture. To adequately use the subtitle based powerless supervision, we propose a LRR based strategy, calledrLRR by acquainting another regularizer with use such frail supervision data. We likewise build up another separation metric learning technique ASML utilizing feeble supervision data to look for a discriminant Mahalanobis separation metric. Two fondness networks can be gotten from rLRR and ASML, separately. In addition, we further breaker the two fondness networks and moreover propose an iterative plan for face naming in light of the combined liking framework. The analyses led on an engineered dataset unmistakably exhibit the viability of the new regularizer in rLRR. In the analyses on two testing certifiable datasets (i.e., the Soccer player dataset and the Labeled Yahoo! News dataset), our rLRR outflanks LRR, and our ASML is superior to anything the current separation metric learning strategy MildML. Besides, our proposed rLRRml beats rLRR and ASML, and also a few best in class benchmark calculations. To further enhance the face naming exhibitions, we plan to augment our rLRR later on by also joining the 1 -standard based regularizer and utilizing different misfortunes when outlining new regularizers. We will likewise concentrate how to consequently focus the ideal parameters for our strategies later on.
[2] A graph based approach for naming faces in news photos.

[3] Robust subspace segmentation by low-rank representation.

[4] Cross-media alignment of names and faces

[5] A computational model for visual selection

[6] Summed area table for texture mapping

[7] A model of saliency based visual attention for rapid scene analysis

[8] Irrelevant feature and subset selection problem

\section{References}

[1] Robust real-time face detection 